\title{
Effect of an columnar defect on vortex configuration in a superconducting mesoscopic sample
}

\author{
J. Barba-Ortega \\ Departamento de Física, Universidade Federal de Pernambuco, 50670-901, Recife, Brasil \\ Ariel Becerra \\ Grupo Integrar, Departamento de Física, Universidad de Pamplona, Colombia \\ J.D. González \\ Grupo de Investigacion en Teoria de la Materia Condensada, \\ Universidad del Magdalena, A.A. 731, Santa Marta, Colombia \\ (Received on 6 July, 2009)
}

\begin{abstract}
In this work we investigate the vortex dynamics in a square mesoscopic superconducting cylinder in the presence of an applied magnetic field parallel to its axis. The rectangular cross-section of the sample is $L^{2}$ and an engineered columnar defect of area $d^{2}$ at the center is taken into account; $L=12 \xi(0)$ for all simulations while $d$ varies discretely from $1 \xi(0)$ to $10 \xi(0)$. We study the magnetization and the vortex configuration, increasing the magnetic field from zero to the normal state field. We found that for $d \geq 7 \xi(0)$ no vortices in the superconductor area are possible. Also, if the size of the defect is reduced, the nucleation fields decrease.
\end{abstract}

Keywords: Ginzburg-Landau, columnar defect, Mesoscopics samples, Superconducting

\section{INTRODUCTION}

The progress of nanofabrication technologies during the last years has resulted in an increased interest in the study of superconducting properties of mesoscopic samples. For type II superconductors the magnetic field, in the so called mixed state, penetrates in the form of vortices, which carry a flux quantum, and form a triangular lattice. For mesoscopic samples, i.e. for a sample of the order of penetration depth, the superconducting properties such as the critical fields, the critical current, the vortex lattice and the vortex itself, can present new and very interesting properties, as for example an increase in the critical field and giant vortices carrying more than one quantum flux $[1,2]$, the effect of defects on two and three dimensional samples on vortex configurations were studied by [5-8]. Several phenomenological theories have been developed during the decades of research in superconductivity. The London theory, the Ginzburg - Landau theory and its time dependent extension, known as Time Dependent Ginzburg - Landau (TDGL) theory have been widely used during the years of research into superconductivity $[3,4]$. In previous work, we have studied the properties of mesoscopic superconducting samples surrounded by different metallic materials using TDGL equations [9-11]. We numerically solve the nonlinear TDGL equations to study vortex dynamics in a mesoscopic type II cylinder superconductor containing one square defect in the presence of an external field applied perpendicular to the surfaces. We analyze the defect size on the vortex configuration, magnetization curves and vortex number. It is assumed that the inner defect edge is in contact with a vacuum. The dynamics of different vortex states and the magnetization curves are studied as a function of the external magnetic field.

\section{THEORETICAL FORMALISM}

Time dependent Ginzburg Landau equations in a zero electric potential gauge and in normalized units lead to the following mathematical problem for the order parameter $\psi$ and the vector potential $\mathbf{A}[12,13]$ :

$$
\begin{aligned}
& \frac{\partial \psi}{\partial t}=-\frac{1}{\eta}\left[(-i \nabla-\mathbf{A})^{2} \psi+(1-T)\left(|\psi|^{2}-1\right) \psi\right] \\
& \frac{\partial \mathbf{A}}{\partial t}=(1-T) \operatorname{Re}\left[\psi^{*}(-i \nabla-\mathbf{A}) \psi\right]-\kappa^{2} \nabla \times \nabla \times \mathbf{A}
\end{aligned}
$$

Where lengths have been scaled in units of $\xi(0)$, time in units of $t_{0}=\pi \hbar /\left(96 K_{B} T_{C}\right)$, A units of $H_{C 2}(0) \xi(0)$, temperatures in units of $T_{C}$.

We used $U \psi$ method [4] for solve the TDGL equations in a discrete grid. Complex link variables $\mathcal{U}^{x}$ and $\mathcal{U}^{y}$ are introduced to preserve the gauge-invariant properties of the discretized equations. $\mathcal{U}^{x}$ and $\mathcal{U}^{y}$ are related to A by:.

$$
\begin{gathered}
\mathcal{U}^{x}(x, y, t)=\exp \left(-i \int_{x_{0}}^{x} A_{x}(\xi, y, t) d \xi\right) \\
\mathcal{U}^{y}(x, y, t)=\exp \left(-i \int_{y_{0}}^{y} A_{y}(x, \eta, t) d \eta\right)
\end{gathered}
$$

The link variable method is used since a better numerical convergence is obtain in high magnetic fields [12]. The TDGL equations 1 and 2 can be written in the following form:

$$
\begin{gathered}
\frac{\partial \psi}{\partial t}=\overline{\mathcal{U}}_{x} \frac{\partial^{2}\left(\mathcal{U}_{x} \psi\right)}{\partial x^{2}}+\overline{\mathcal{U}}_{y} \frac{\partial^{2}\left(\mathcal{U}_{y} \psi\right)}{\partial y^{2}} \\
+(1-T) \psi\left(1-|\psi|^{2}\right) \\
\mathbf{J}_{\mathbf{s} \alpha}=(1-T) \operatorname{Im}\left[\overline{\mathcal{U}}_{\alpha} \bar{\psi} \frac{\partial\left(\mathcal{U}_{\alpha} \psi\right)}{\partial \alpha}\right]
\end{gathered}
$$

where $\alpha=(x, y)$, and Im indicates the imaginary part. We used this method to obtain our results. The outline of this 
simulation procedure is as follows: the sample is divided in a rectangular mesh consisting of $N_{x} \times N_{y}$ cells, with mesh spacing $a_{x} \times a_{y}$. To derive the discrete equations let us define by $x_{i}=(i-1) a_{x}, y_{i}=(i-1) a_{y}$, an arbitrary vertex point in the mesh and:

$$
\begin{gathered}
U_{i, j}^{x}=\overline{\mathcal{U}}_{i, j}^{x} \mathcal{U}_{i+1, j}^{x}=\exp \left(-i \int_{x_{i}}^{x_{i}+1} A_{x}\left(\xi, y_{i}\right) d \xi\right) \\
U_{i, j}^{y}=\overline{\mathcal{U}}_{i, j}^{y} \mathcal{U}_{i, j+1}^{x}=\exp \left(-i \int_{y_{j}}^{y_{j}+1} A_{y}\left(x_{i}, \eta\right) d \eta\right) \\
L_{i, j}=U_{i, j}^{x} U_{i+1, j}^{y} \bar{U}_{i, j+1}^{x} \bar{U}_{i, j}^{y}=\exp \left(-i a_{x} a_{y} H_{e}\right)
\end{gathered}
$$

Then the discretized version of the TDGL equations maintaining second order accuracy in space are gyven by:

$$
\begin{gathered}
\frac{\partial \psi_{i, j}}{\partial t}=\frac{U_{i, j}^{x} \psi_{i, j}-2 \psi_{i, j}+\bar{U}_{i-1, j}^{x} \psi_{i-1, j}}{\eta a_{x}^{2}}+ \\
\frac{U_{i, j}^{y} \psi_{i, j+1}-2 \psi_{i, j}+\bar{U}_{i, j-1}^{y} \psi_{i, j-1}}{\eta_{y}^{2}} \\
-\frac{1-T}{\eta}\left(\bar{\psi}_{i, j} \psi_{i, j}-1\right) \psi_{i, j}+\tilde{f}_{i, j} \\
\frac{\partial U_{i, j}^{x}}{\partial t}=-i(1-T) U_{i, j}^{x} \operatorname{Im}\left(\bar{\psi}_{i, j} U_{i, j}^{x} \psi_{i+1, j}\right)- \\
\frac{\partial U_{i, j}^{y}}{\partial t}=-i(1-T) U_{i, j}^{y} \operatorname{Im}\left(\bar{\psi}_{i, j} U_{i, j}^{x} \psi_{i, j+1}^{x}\left(\bar{L}_{i, j-1} L_{i, j}-1\right)-\right. \\
\frac{\kappa^{2}}{a_{x}^{2}} U_{i, j}^{y}\left(\bar{L}_{i, j} L_{i-1, j}-1\right)
\end{gathered}
$$

The discretized open boundary conditions are:

$$
\begin{gathered}
\psi_{1, j}=\psi_{2, j} U_{1, j}^{x} \\
\psi_{N_{x}+1, j}=\psi_{N_{x}, j} \bar{U}_{N_{x}, j}^{x}
\end{gathered}
$$

We use the simple Euler method with $10^{7}$ steps, $\Delta t=0.0015$, spacing $a_{x}=a_{y}=0.25 . \vec{H}_{e}$ is increased linearly with time from 0 to 1 , with small intervals of $\Delta H=10^{-7}, \kappa=5, T=0$ for all simulations.

The procedure consists of beginning of certain value of the applied magnetic field $H_{e}$ and the order parameter $\psi(r, t=0)$, i.e. the variables are homogeneously initialized to a perfect Meissner state $\psi(t=0)=1, \mathbf{A}(t=0)=0$ (for every point in the domain). The stationary state found for a fixed $\mathrm{He}$ is used then as initial condition for the next field value $H+\Delta H$, using small increments of $\Delta H$. The applied magnetic field is increased smoothly from zero to a value where the superconductivity will be destroyed completely. For each applied field we follow the temporary evolution of the magnetic induction and of the superconducting order parameter to obtain a stationary solution.

We considered a mesoscopic superconducting cylinder with a rectangular cross section $L^{2}$ with an engineered columnar defect of area $d^{2}$ at its center. The TDGL equations are used upon taking the magnetic field and the order parameter invariant along $z$-direction led to bi-dimensional

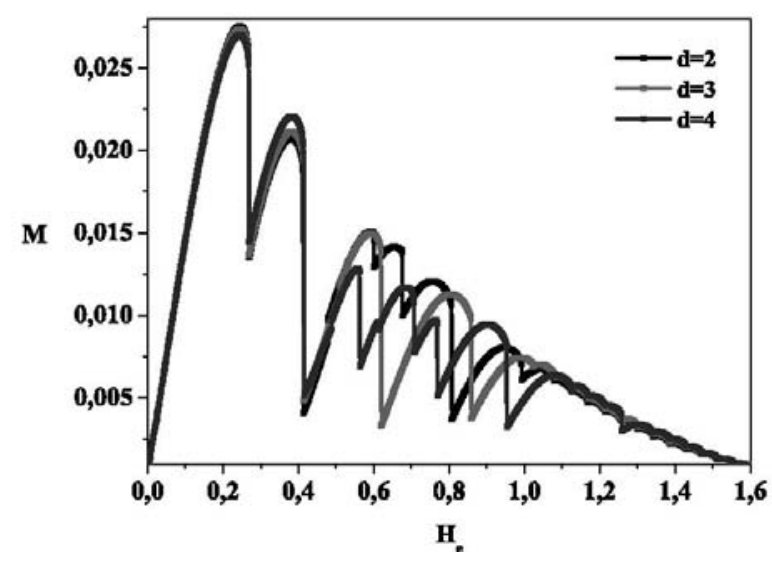

Fig. 1. Magnetization curves as function of the applied external field for a cylinder of $L=12 \xi(0)$, with a centered defect with $d / \xi(0)=2,3,4$.

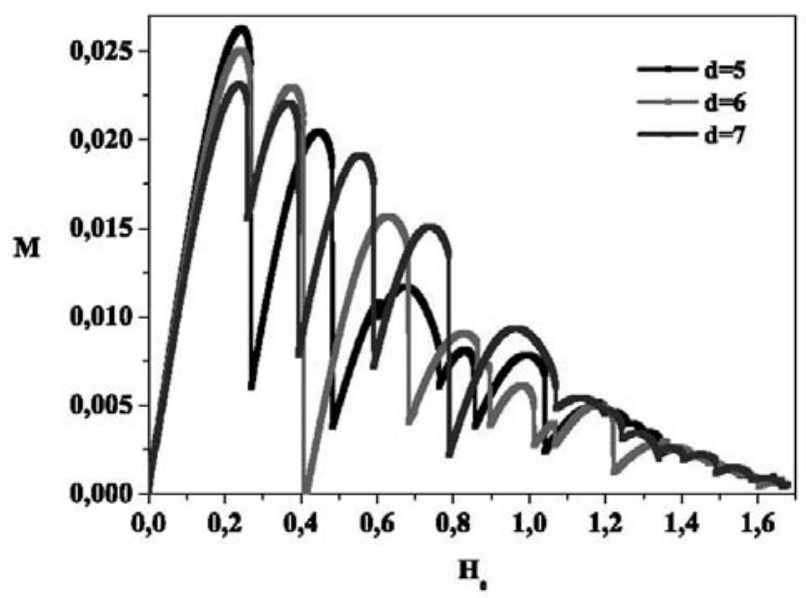

Fig. 2. Magnetization curves as function of the applied external field for a cylinder of $L=12 \xi(0)$, with a centered defect with $d / \xi(0)=5,6,7$.

numerical treatment. The superconducting wave function satisfies the boundary conditions $\widehat{n} \cdot(-i \nabla-\mathbf{A}) \psi=0$, where $\widehat{n}$ denotes the normal vector to the superconductor - vacuum interface, and the boundary conditions for $\mathbf{A}$, namely that $B_{z}=\widehat{e_{z}} \cdot \nabla \times \mathbf{A}$ at the external surface must equal $\mathbf{H}_{\mathbf{e}}$, the applied field. The defect has the following dimensions: $d=1 \xi(0)$ to $d=10 \xi(0)$ and $L=12 \xi(0)$.

\section{RESULTS}

The results for the magnetization curves $M$ as a function of the applied external field $H_{e}$ are depicted in Figs. 1,2,3. The magnetization curves exhibit a series of discontinuities; each of these discontinuities signals a vortex entrance in the sample. As is well known we can appreciate that if we reduce the size of the defect, $H_{c 2}$ remains constant $H_{c 2}=1.6$ for $d=2,3,4$ and increases $H_{c 2}=1.7$ for $d=5,6,7$ and $H_{c 2}=2.0,2.5,4.0$ for $d=8,9,10$, with vorticity $N=28$ for 


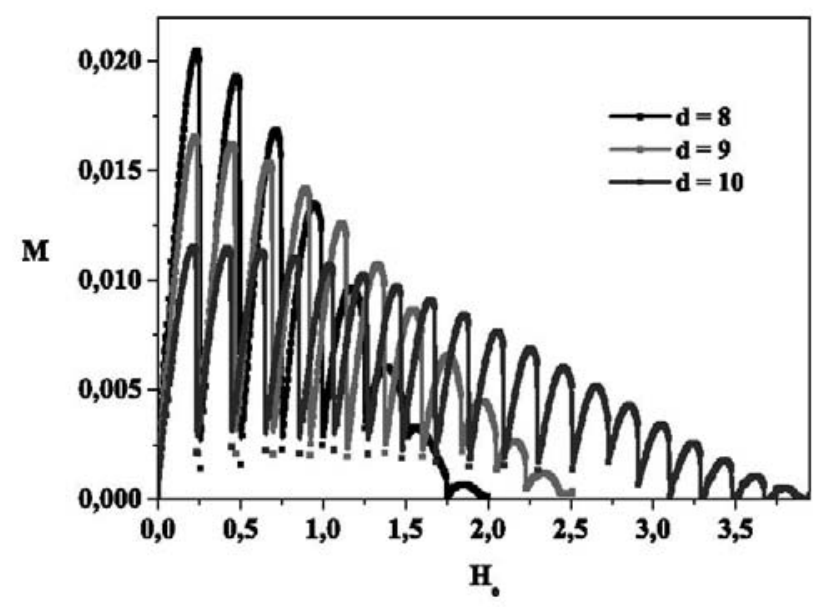

Fig. 3. Magnetization curves as function of the applied external field for a cylinder of $L=12 \xi(0)$, with a centered defect with $d / \xi(0)=8,9,10$.
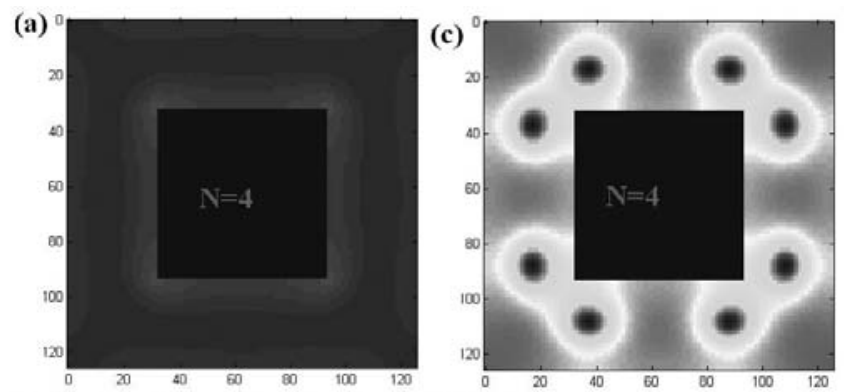

(b)
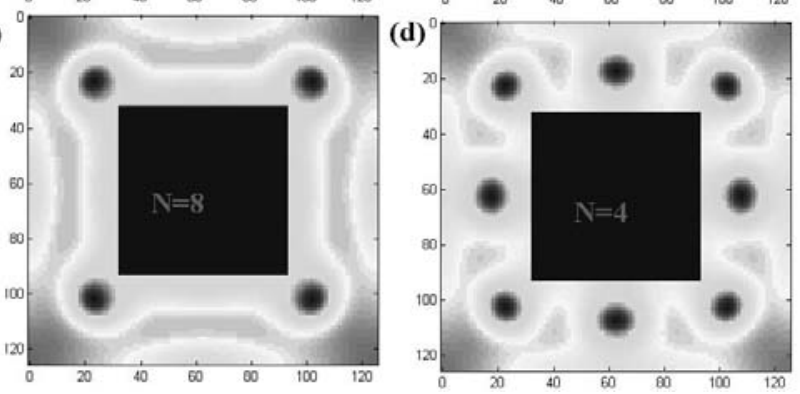

Fig. 4. Snapshot of the vortex configuration for $d=6 \xi(0)$ and (a) $N=4$, (b), (c) and (d) $N=12$ at

$H_{e} / H_{c 2}(0)=0.40,0.840,0.845,0.847$ respectively, blue and red regions represents values of the modulus of the order parameter from 0 to 1 .

$d=1,2,3,4, N=30$ for $d=5,6, N=32$ for $d=7,8, N=44$ for $d=9$ and $N=72$ for $d=10$. The vortex number in the sample increases as defect size increases. For a defect $d \geq 7$, the sample has ring superconductor behavior, $H_{c 2}$ increase and no vortices are possible in the superconductor region, $H_{c 1}$ remains constant for all samples. We do not include the sample with $d=1$ in order for the figure to be better visualized.

The magnitude of the order parameter $|\psi|$ is plotted in Figures 4 for a sample with $d=6 \xi(0)$. In Figs. $4(\mathrm{a}-\mathrm{d})$, values of the order parameter close to zero are given by blue regions and close to 1 by red regions.

In Fig. 4(a) the first four vortices will be located in the

defect. Although they are not visible in the contour plot of the magnitude of order parameter, there is a change in the phase around each hole equal to $\Delta \phi=8 \pi$. The phase allows us to determine the number of vortices in a given region, by counting the phase variation in a closed path around this region. If the vorticity in this region is $N$, then the phase changes by $\Delta \phi=2 \pi N$ [13]. By increasing the magnetic field eight more vortices appear in the sample, as shown in Fig 4(b). They are localized in the superconductor region and goes to the defect corner. Then, in Fig. 4(c), eight vortices are situated in the hole and four vortices are in the superconducting region, while four vortices are going out of the defect. In Fig 4(d), with $N=12$, four vortices remain in the defect hole and eight in the superconducting region. All the states are not stationary states.

In the next tables we shown the magnetic field $H_{e}$ with the pair of vorticities - inside and outside the defect.

$\begin{array}{cccc}\text { He } & \text { Ninside } & \text { Noutside } & \text { Ntotal } \\ 0.20 & 0 & 0 & 0 \\ 0.24 & 0 & 2 & 2 \\ 0.42 & 0 & 4 & 4 \\ 0.60 & 0 & 6 & 6 \\ 0.64 & 0 & 8 & 8 \\ 0.72 & 0 & 10 & 10 \\ 0.98 & 0 & 12 & 12\end{array}$

TABLE I: Vorticies inside and outside the defect for a sample $L=$ $12 \xi(0)$ and $d=1 \xi(0)$

$\begin{array}{cccc}\text { He } & \text { Ninside } & \text { Noutside } & \text { Ntotal } \\ 0.20 & 0 & 0 & 0 \\ 0.24 & 0 & 2 & 2 \\ 0.39 & 0 & 4 & 4 \\ 0.57 & 2 & 4 & 6 \\ 0.70 & 4 & 6 & 10 \\ 0.95 & 6 & 8 & 14 \\ 1.00 & 6 & 8 & 14\end{array}$

TABLE II: Vorticies inside and outside the defect for a sample $L=$ $12 \xi(0)$ and $d=4 \xi(0)$

$\begin{array}{cccc}\text { He } & \text { Ninside } & \text { Noutside } & \text { Ntotal } \\ 0.20 & 4 & 0 & 4 \\ 0.40 & 4 & 2 & 6 \\ 0.45 & 4 & 4 & 8 \\ 0.50 & 4 & 6 & 10 \\ 0.840 & 4 & 8 & 12 \\ 0.845 & 8 & 4 & 12 \\ 0.847 & 4 & 8 & 12\end{array}$

TABLE III: Vorticies inside and outside the defect for a sample $L=$ $12 \xi(0)$ and $d=6 \xi(0)$

The magnitude of the order parameter is plotted in Figure 5 for a sample with $d=1$ for (a) $H_{e}=0.2, N=0$. (b) $H_{e}=$ 0.72, $N=10$. (c) $H_{e}=0.9, N=12, d=8$ for (d) $H_{e}=$ 0,86, $N=12, d=9$ for (e) $H_{e}=1.04, N=16$ and $d=$ 10 for (f) $H_{e}=1.11$. $N=18$. We can observe a sequence of transitions as the magnetic external field increases. The 


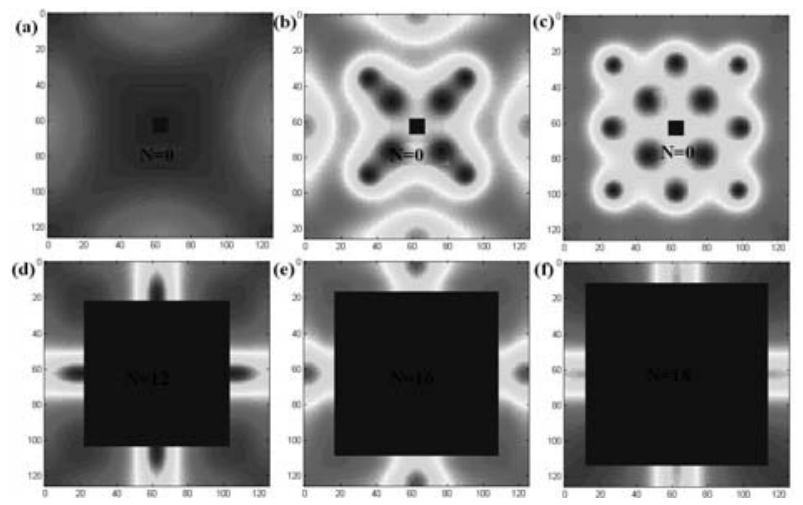

Fig. 5. Snapshot of the vortex configuration for $d=1 \xi(0)$ for (a) $H_{e}=0.2 H_{c 2}(0), N=0$. (b) $H_{e}=0.72 H_{c 2}(0), N=10$. (c) $H_{e}=0.9 H_{c 2}(0), N=12$ and $d=8 \xi(0)$ for $(\mathrm{d}) H_{e}=0,86 H_{c 2}(0)$, $N=12 . d=9 \xi(0)$ for (e) $H_{e}=1.04 H_{c 2}(0), N=16$ and $d=10 \xi(0)$ for (f) $H_{e}=1.11 H_{c 2}(0) . N=18$.

sample with $d=1 \xi(0)$ does not allow vortices in the defect, due to size restrictions. $d>7 \xi(0)$ does not present vortices in the superconductor area.

\section{CONCLUSIONS}

We theoretically calculated the spatial distribution of the vortices in a cylindrical mesoscopic superconductor with one square defect. All the work done here is for an infinitely long sample with mesoscopic square cross section with size $L=12 \xi(0)$. The presence of the defect affects the vortex distribution, as well as the vortex entry. We observed a sequence of transitions as the magnetic external field increases. Samples with defects $d \geq 7$ do not present vortices in the superconductor area. Our results also show that, as we reduce the size of the defect, the nucleation field decreases. The main goal of this work is to determine the limit of the occurrence of square ring superconductor behavior.

\section{ACKNOWLEDGEMENTS}

The authors thank to Helen Cater for useful discussions.
[1] L. Chibotaru, A. Celeumans, V. Bruyndoncx, V. Moshchalkov, Nature (London) 348, 833 (2000).

[2] B. Baelus, F. Peeters and V. Schweigert Phys. Rev. B 61, 9734, (2000).

[3] G. C. Buscaglia, C. Bolech and A. López, Connectivity and Superdonductivity, J. Berger and J. Rubinstein (Eds.) Springer, 2000.

[4] D. Gropp, H. Kaper, G. Leaf, D. Levine, M. Palumbo and V. Vinokur, J. Comput. Phys. 123, 254, (1996).

[5] R. de C. Romaguera, Mauro M. Doria and F. M. Peeters, Phys. Rev. B 75, 184525, (2007). Phys. Rev. B 75, 064505, (2007).

[6] M. Doria, S. C. Andrade, Phys. Rev. B 60, 13164, (1997).

[7] E. Sardella, R.A. Freire, P. N. Lisboa-Filho, Physica C 421, $41,(2005)$
[8] E. Sardella, A. L. Malvezzi, P. N. Lisboa-Filho, Phys. Rev. B 74, 014512, (2006), Phys. Rev. B 77, 104508, (2008). Physica. B 403, 1494, (2008).

[9] José J. Barba, Clécio C. Souza, L. Cabral and J. Albino Aguiar, Physica C 468, 718 (2008).

[10] J. Barba-Ortega and J. Albino Aguiar, Journal of Physics: Conference series 150, 052015 (2009).

[11] J. Barba-Ortega and J. Albino Aguiar, Physica C 469, 754 (2009). Physica C 469, 852, (2009).

[12] R. Kato, Y. Enomoto and S. Maekawa Phys. Rev. B, 44, 6916 (1991).

[13] P. G. de Gennes, Superconductivity of Metals and Alloys, Benjamin, New York 1966. 